\title{
On the dynamic behaviour of the Timoshenko beam finite elements
}

\author{
J N REDDY \\ Department of Mechanical Engineering, Texas A \& M University, College \\ Station, TX 77843-3123, USA \\ e-mail: jnreddy@ reddy20.tamu.edu
}

MS received 15 November 1998; revised 13 January 1999

\begin{abstract}
First, various finite element models of the Timoshenko beam theory for static analysis are reviewed, and a novel derivation of the $4 \times 4$ stiffness matrix (for the pure bending case) of the superconvergent finite element model for static problems is presented using two alternative approaches: (1) assumed-strain finite element model of the conventional Timoshenko beam theory, and (2) assumed-displacement finite element model of a modified Timoshenko beam theory. Next, dynamic versions of various finite element models are discussed. Numerical results for natural frequencies of simply supported beams are presented to evaluate various Timoshenko beam finite elements. It is found that the reduced integration element predicts the natural frequencies accurately, provided a sufficient number of elements is used.
\end{abstract}

Keywords. Timoshenko beam finite elements; superconvergent element; dynamic behaviour; natural frequencies; assumed strain-displacement formulation; interdependent interpolation; reduced integration elements.

\section{Introduction}

The development of structural and finite element models of the Timoshenko beam theory (i.e., include transverse shear deformation in the stiffness matrix) has been the subject of numerous papers in the literature. The exact, $4 \times 4$ stiffness matrix of the Timoshenko beam is derived either using the methods of structural analysis (see Gere \& Weaver 1965, Przemieniecki 1968, Reddy et al 1997) or finite element formulations (see Friedman and Kosmatka, 1993, Reddy 1997); Most papers dealing with finite element models of the Timoshenko beam theory focus on alleviating shear and membrane locking by selecting proper interpolations of the deflections and shear strain and/or the displacement or assumed strain-displacement method. Most of these approaches did not result in the twonode superconvergent element (i.e. element that gives exact values of the generalized 
displacements at the nodes) with four degrees of freedom per element, as is the case with the Euler-Bernoulli beam element.

The shear locking is due to the inconsistency of the interpolation used for $w$ and $\phi$, or equivalently, not satisfying the requirement that the shear strain $\gamma_{x z}=(\mathrm{d} w / \mathrm{d} x)+\phi$ is element-wise constant for element-wise constant values of $E I$. Often, the Timoshenko finite element models are based on equal interpolation of $w$ and $\phi$ and use reduced-order integration to evaluate the stiffness coefficients associated with the transverse shear strain and full integration for all other coefficients. Others have used so-called consistent interpolation based on the the recovery of correct constraints in the thick beam limit (Prathap \& Bhashyam 1982; Babu \& Prathap 1986; Prathap \& Babu 1986; Shi \& Voyiadjis 1991; Rakowski 1991; Reddy 1993). Although such elements do not experience locking, they do not lead to the two-node superconvergent element. Friedman \& Kosmatka (1993) and Reddy (1997) and Reddy et al (1997) have independently developed the two-node superconvergent element using the exact solution of the homogeneous form of the Timoshenko beam equations. Hermite cubic interpolation of $w$ and interdependent quadratic interpolation of $\phi$ was used in developing the element that has the superconvergence character for static problems. The mass matrices for various elements are also presented. Friedman \& Kosmatka (1993) discussed the dynamic version of the interdependent interpolation Timoshenko beam element but did not realize that it would not represent the pure shear frequencies accurately.

The objective of the present paper is two-fold: (1) to present alternative derivations of the stiffness matrices associated with the reduced integration finite element and superconvergent finite element, and (2) to extend the elements to the dynamic case and evaluate their behaviour. Two separate approaches, not using the exact solution, are presented for the development of the stiffness matrix of the superconvergent finite element. The first one is based on the assumed strain-displacement approach, and the second one on the two-component form of the Timoshenko beam theory. Modifications to the stiffness and mass matrices are also suggested that yield more accurate pure shear frequencies.

\section{Theoretical formulation}

The displacement field of the Timoshenko beam theory for the pure bending case is

$$
u_{1}(x, z)=z \phi(x), \quad u_{2}=0, \quad u_{3}(x, z)=w(x),
$$

where $w$ is the transverse deflection and $\phi_{x}$ the rotation of a transverse normal line about the $y$ axis. The strains and stresses of the Timoshenko beam theory are

$$
\varepsilon_{x x}=z \frac{\mathrm{d} \phi_{x}}{\mathrm{~d} x} \equiv z \kappa_{x x}, \quad \gamma_{x z}=\phi_{x}+\frac{\mathrm{d} w}{\mathrm{~d} x}, \quad \sigma_{x x}=E \varepsilon_{x x}, \quad \sigma_{x z}=G \gamma_{x z} .
$$

The equilibrium equations of the beam are

$$
\begin{aligned}
& -\frac{\mathrm{d}}{\mathrm{d} x}\left(E I \frac{\mathrm{d} \phi_{x}}{\mathrm{~d} x}\right)+G A K_{s}\left(\phi_{x}+\frac{\mathrm{d} w}{\mathrm{~d} x}\right)=0 \\
& -\frac{\mathrm{d}}{\mathrm{d} x}\left[G A K_{s}\left(\phi_{x}+\frac{\mathrm{d} w}{\mathrm{~d} x}\right)\right]=q(x)
\end{aligned}
$$


where $q(x)$ is the distributed transverse load, $E$ Young's modulus, $G$ the shear modulus, $A$ the area of cross section, $I$ the moment of inertia, and $K_{S}$ the shear correction factor.

\section{Displacement finite element models}

\subsection{The general model}

The displacement finite element model of the Timoshenko beam theory is constructed using the principle of minimum total potential energy, or equivalently, using the weak form

$$
\begin{aligned}
0= & \int_{x_{a}}^{x_{b}}\left[E I \frac{\mathrm{d} \delta \phi_{x}}{\mathrm{~d} x} \frac{\mathrm{d} \phi_{x}}{\mathrm{~d} x}+G A K_{s}\left(\delta \phi_{x}+\frac{\mathrm{d} \delta w}{\mathrm{~d} x}\right)\left(\phi_{x}+\frac{\mathrm{d} w}{\mathrm{~d} x}\right)\right] \mathrm{d} x \\
& -\int_{x_{a}}^{x_{b}} q(x) \delta w \mathrm{~d} x-V_{a} \delta w\left(x_{a}\right)-V_{b} \delta w\left(x_{b}\right) \\
& -M_{a} \delta \phi_{x}\left(x_{a}\right)-M_{b} \delta \phi_{x}\left(x_{b}\right)
\end{aligned}
$$

and (see figure 1)

$$
\begin{aligned}
& V_{a} \equiv-Q\left(x_{a}\right)=-\left[G A K_{s}\left(\frac{\mathrm{d} w}{\mathrm{~d} x}+\phi_{x}\right)\right]_{x=x_{a}}, \\
& M_{a} \equiv-M\left(x_{a}\right)=-\left[E I \frac{\mathrm{d} \phi_{x}}{\mathrm{~d} x}\right]_{x=x_{a}}, \\
& V_{b} \equiv Q\left(x_{b}\right)=\left[G A K_{s}\left(\frac{\mathrm{d} w}{\mathrm{~d} x}+\phi_{x}\right)\right]_{x=x_{b}}, \\
& M_{b} \equiv M\left(x_{b}\right)=\left[E I \frac{\mathrm{d} \phi_{x}}{\mathrm{~d} x}\right]_{x=x_{b}}
\end{aligned}
$$

Suppose that $w$ and $\phi_{x}$ are approximated as

$$
w(x) \approx \sum_{j=1}^{m} \psi_{j}^{(1)} W_{j}, \quad \phi_{x}(x) \approx \sum_{j=1}^{n} \psi_{j}^{(2)} \Phi_{j},
$$

where $\left(W_{j}, \Phi_{j}\right)$ are the nodal values of $\left(w, \phi_{x}\right)$ and $\psi_{j}^{(\alpha)}(x)(\alpha=1,2)$ are the associated interpolation functions. Substitution of (7) for $w$ and $\phi_{x}$, and $\delta w=\psi_{i}^{(1)}$ and $\delta \phi_{x}=\psi_{i}^{(2)}$ into (5) yields the finite element model

$$
\left[\begin{array}{cc}
{\left[K^{11}\right]} & {\left[K^{12}\right]} \\
{\left[K^{12}\right]^{\mathrm{T}}} & {\left[K^{22}\right]}
\end{array}\right]\left\{\begin{array}{c}
\{W\} \\
\{\Phi\}
\end{array}\right\}=\left\{\begin{array}{l}
\left\{F^{1}\right\} \\
\left\{F^{2}\right\}
\end{array}\right\},
$$

where

$$
\begin{aligned}
& K_{i j}^{11}=\int_{x_{a}}^{x_{b}} K_{s} G A \frac{\mathrm{d} \psi_{i}^{(1)}}{\mathrm{d} x} \frac{\mathrm{d} \psi_{j}^{(1)}}{\mathrm{d} x} \mathrm{~d} x, \\
& K_{i j}^{12}=\int_{x_{a}}^{x_{b}} K_{s} G A \frac{\mathrm{d} \psi_{i}^{(1)}}{\mathrm{d} x} \psi_{j}^{(2)} \mathrm{d} x,
\end{aligned}
$$



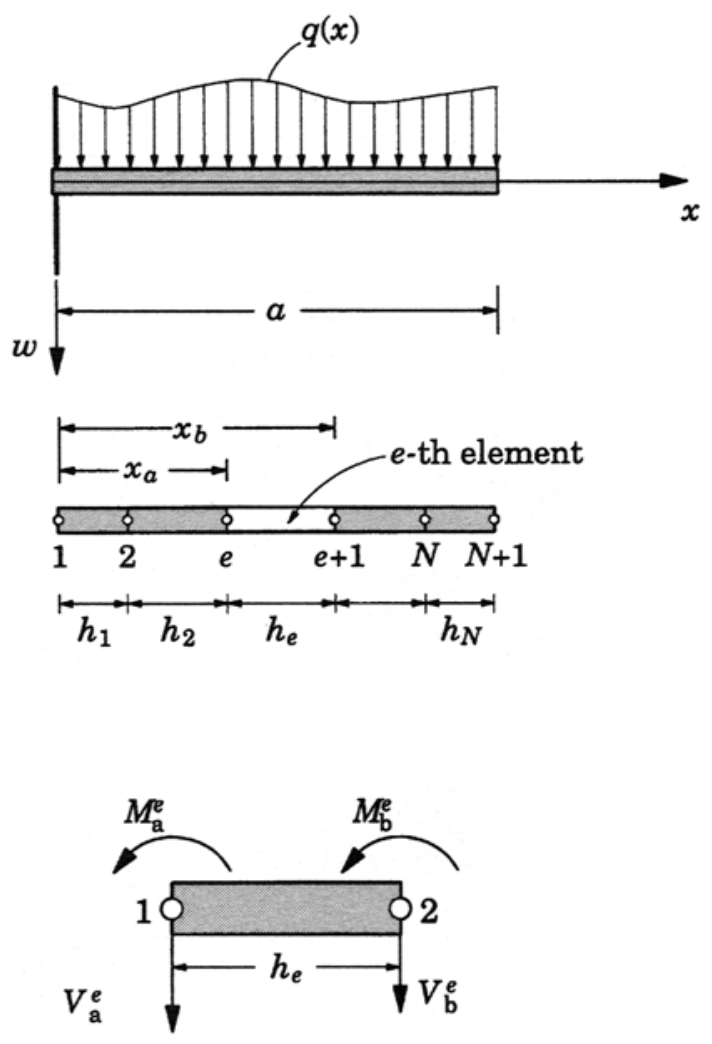

Secondary variables

Figure 1. Typical finite element with force degrees of freedom.

$$
\begin{aligned}
K_{i j}^{22} & =\int_{x_{a}}^{x_{b}}\left(E I \frac{\mathrm{d} \psi_{i}^{(2)} \mathrm{d} \psi_{j}^{(2)}}{\mathrm{d} x}+K_{s} G A \psi_{i}^{(2)} \psi_{j}^{(2)}\right) \mathrm{d} x, \\
F_{i}^{1} & =\int_{x_{a}}^{x_{b}} \psi_{i}^{(1)} q \mathrm{~d} x+V_{a} \psi_{i}^{(1)}\left(x_{a}\right)+V_{b} \psi_{i}^{(1)}\left(x_{b}\right), \\
F_{i}^{2} & =M_{a} \psi_{i}^{(2)}\left(x_{a}\right)+M_{b} \psi_{i}^{(2)}\left(x_{b}\right) .
\end{aligned}
$$

\subsection{Reduced integration element (RIE)}

For a linear interpolation of $w$ and $\phi_{x}$ and exact evaluation of the integrals of (9), (8) takes the form

where

$$
\left(\frac{2 E I}{\mu_{0} h^{3}}\right)\left[\begin{array}{cccc}
6 & -3 h & -6 & -3 h \\
-3 h & 2 h^{2} \lambda & 3 h & h^{2} \xi \\
-6 & 3 h & 6 & 3 h \\
-3 h & h^{2} \xi & 3 h & 2 h^{2} \lambda
\end{array}\right]\left\{\begin{array}{c}
W_{1} \\
\Phi_{1} \\
W_{2} \\
\Phi_{2}
\end{array}\right\}=\left\{\begin{array}{c}
q_{1}^{(1)} \\
0 \\
q_{2}^{(1)} \\
0
\end{array}\right\}+\left\{\begin{array}{c}
V_{a} \\
M_{a} \\
V_{b} \\
M_{b}
\end{array}\right\}
$$

$$
q_{i}^{(1)}=\int_{x_{a}}^{x_{b}} \psi_{i}^{(1)} q \mathrm{~d} x, \quad(i=1,2)
$$




$$
\Omega=\frac{E I}{G A K_{s} h^{2}}, \quad \mu_{0}=12 \Omega, \quad \xi=1-6 \Omega, \quad \lambda=1+3 \Omega .
$$

In the thin beam limit, i.e., $\Omega \rightarrow 0$, the first and third equations of (10) imply the following relation among $\left(W_{1}, W_{2}, \Phi_{1}, \Phi_{2}\right)$ :

$$
\left(\frac{\Phi_{1}+\Phi_{2}}{2}\right)+\left(\frac{W_{2}-W_{1}}{h}\right)=0
$$

which is equivalent to the Kirchhoff constraint $\phi_{x}+\mathrm{d} w / \mathrm{d} x=0$ (or shear strain $\gamma_{x z}=0$ ). The second and fourth equations of (10), in view of (12), yield the constraint

$$
\left(\Phi_{1}-\Phi_{2}\right) / h=0 .
$$

This is equivalent to $\mathrm{d} \phi_{x} / \mathrm{d} x=0$, which is an incorrect condition to satisfy as it forces the curvature and hence the bending energy to zero. Thus, (10), in an effort to satisfy the constraints (12) and (13), will yield the trivial solution $W_{1}=W_{2}=\Phi_{1}=\Phi_{2}=0$ (i.e., the element locks).

The Kirchhoff condition (12) suggests that $w$ and $\phi_{x}$ be interpolated such that $\mathrm{d} w / \mathrm{d} x$ is a polynomial of the same order as $\phi_{x}$. If $w$ is approximated using a linear polynomial (a minimum requirement), then $\phi_{x}$ should be a constant. Since the minimum continuity requirement on $\phi_{x}$ is also linear, it follows that $w$ be approximated using a quadratic polynomial. This is a consistent interpolation. Unless the weak form of the Timoshenko beam theory is modified, we have no alternative but to use a quadratic approximation of $w$ and linear for $\phi_{x}$ and use full integration to evaluate the coefficient matrices to obtain an element that does not experience locking. However, if one approximates both $w$ and $\phi_{x}$ with linear polynomials but treats $\phi_{x}$ as a constant in the evaluation of the shear strain, it will also yield the stiffness matrix. This procedure is known in the literature as reduced integration of the shear stiffness. It amounts to evaluating the second term of $K_{i j}^{22}$ in (9) using one-point integration as opposed to two-point integration required to exactly evaluate the integral. The element equations of the reduced integration element are

$$
\begin{aligned}
\left(\frac{2 E I}{\mu_{0} h^{3}}\right) & {\left[\begin{array}{cccc}
6 & -3 h & -6 & -3 h \\
-3 h & h^{2}(1.5+6 \Omega) & 3 h & h^{2}(1.5-6 \Omega) \\
-6 & 3 h & 6 & 3 h \\
-3 h & h^{2}(1.5-6 \Omega) & 3 h & h^{2}(1.5+6 \Omega)
\end{array}\right]\left\{\begin{array}{l}
W_{1} \\
\Phi_{1} \\
W_{2} \\
\Phi_{2}
\end{array}\right\} } \\
= & \left\{\begin{array}{c}
q_{1}^{(1)} \\
0 \\
q_{2}^{(1)} \\
0
\end{array}\right\}+\left\{\begin{array}{c}
V_{a} \\
M_{a} \\
V_{b} \\
M_{b}
\end{array}\right\} .
\end{aligned}
$$

This element is designated as the reduced integration element (RIE) by Reddy (1993). Alternate derivation of the element without using the reduced integration concepts will be presented in the sequel. In the thin beam limit, the element equations reduce to only one constraint, namely the Kirchhoff condition in (12). While the element does not lock, it does not yield exact displacements at the nodes for the static problems, and often a sufficient number of elements is needed to obtain accurate deflections. 


\subsection{Consistent interpolation element (CIE)}

As suggested earlier, if we use a quadratic approximation of $w$ and linear approximation of $\Phi,(8)$ reduces to a $5 \times 5$ system of equations. By eliminating the mid-side degree of freedom associated with $w$, we can reduce the $5 \times 5$ system to the following $4 \times 4$ system of equations (Reddy 1993; Reddy 1999):

$$
\begin{aligned}
\left(\frac{2 E I}{\mu_{0} h^{3}}\right) & {\left[\begin{array}{cccc}
6 & -3 h & -6 & -3 h \\
-3 h & h^{2}(1.5+6 \Omega) & 3 h & h^{2}(1.5-6 \Omega) \\
-6 & 3 h & 6 & 3 h \\
-3 h & h^{2}(1.5-6 \Omega) & 3 h & h^{2}(1.5+6 \Omega)
\end{array}\right]\left\{\begin{array}{l}
W_{1} \\
\Phi_{1} \\
W_{2} \\
\Phi_{2}
\end{array}\right\} } \\
= & \left\{\begin{array}{c}
q_{1}^{(2)}+\frac{1}{2} q_{c}^{(2)} \\
-\frac{1}{8} q_{c}^{(2)} h \\
q_{2}^{(2)}+\frac{1}{2} q_{c}^{(2)} \\
\frac{1}{8} q_{c}^{(2)} h
\end{array}\right\}+\left\{\begin{array}{c}
V_{a} \\
M_{a} \\
V_{b} \\
M_{b}
\end{array}\right\},
\end{aligned}
$$

where

$$
q_{i}^{(2)}=\int_{x_{a}}^{x_{b}} \psi_{i}^{(2)} q \mathrm{~d} x, \quad(i=1,2, \mathrm{c}),
$$

and $\psi_{i}^{(2)}$ are the quadratic interpolation functions. Here the subscript $c$ is used for the centre node of the element. Note that the element has the same stiffness matrix as the reduced integration element but a different load vector. The load vector is equivalent to that of the Euler-Bernoulli beam element. In fact, for constant $q$, the load vector in (15) is identical to that of the Euler-Bernoulli beam element. The elimination of the mid-side deflection degree of freedom is not possible for the dynamic case, as will be apparent in the sequel.

\subsection{Interdependent interpolation element (IIE)}

The next choice of consistent interpolation is to use cubic for $w$ and quadratic for $\phi_{x}$. This will lead to a $7 \times 7$ system of equations. The displacement degrees of freedom associated with the interior nodes (three in total) can again be condensed out, for the static case, to obtain a $4 \times 4$ system of equations. Here we will not consider it further. Instead, we consider the Hermite cubic interpolation of $w$ and a related quadratic approximation of $\phi_{x}$. These sets of interpolation functions were derived by Reddy (1997) using the exact solution of (3) and (4) for $q=0$. The resulting finite element is termed the interdependent interpolation element (IIE).

To develop the interdependent interpolation element, we assume an approximation of the form

$$
\begin{aligned}
w(x) & \approx \sum_{j=1}^{m} \varphi_{j}^{(1)} \Delta_{j}, \quad \phi_{x}(x) \approx \sum_{j=1}^{n} \varphi_{j}^{(2)} \Delta_{j}, \\
\Delta_{1} & =W_{1}, \quad \Delta_{2}=\Phi_{1}, \quad \Delta_{3}=W_{2}, \quad \Delta_{4}=\Phi_{2},
\end{aligned}
$$


where $\varphi_{i}^{(1)}$ and $\varphi_{i}^{(2)}$ are the approximation functions.

$$
\begin{aligned}
& \varphi_{1}^{(1)}=(1 / \mu)\left[\mu-12 \Omega \eta-(3-2 \eta) \eta^{2}\right], \\
& \varphi_{2}^{(1)}=-(h / \mu)\left[(1-\eta)^{2} \eta+6 \Omega(1-\eta) \eta\right], \\
& \varphi_{3}^{(1)}=(1 / \mu)\left[(3-2 \eta) \eta^{2}+12 \Omega \eta\right], \\
& \varphi_{4}^{(1)}=(h / \mu)\left[(1-\eta) \eta^{2}+6 \Omega(1-\eta) \eta\right], \\
& \varphi_{1}^{(2)}=(6 / h \mu)(1-\eta) \eta \\
& \varphi_{2}^{(2)}=(1 / \mu)\left(\mu-4 \eta+3 \eta^{2}-12 \Omega \eta\right), \\
& \varphi_{3}^{(2)}=-(6 / h \mu)(1-\eta) \eta \\
& \varphi_{4}^{(2)}=(1 / \mu)\left(3 \eta^{2}-2 \eta+12 \Omega \eta\right) .
\end{aligned}
$$

Here $\eta$ is the nondimensional local coordinate

$$
\eta=\left(x-x_{a}\right) / h, \quad \mu=1+12 \Omega \text {. }
$$

When $\Omega=0, \varphi_{i}^{(1)}$ reduces to the usual Hermite interpolation functions $\varphi_{i}$ and $\varphi_{i}^{(2)}$ to $-\mathrm{d} \varphi_{i} / \mathrm{d} x$. Substitution of (17) into (5) yields the finite element model,

$$
[K]\{\Delta\}=\{q\}+\{Q\},
$$

where

$$
\begin{aligned}
K_{i j} & =\int_{x_{a}}^{x_{b}}\left[E I \frac{\mathrm{d} \varphi_{i}^{(2)} \mathrm{d} \varphi_{j}^{(2)}}{\mathrm{d} x}+G A K_{s}\left(\varphi_{i}^{(2)}+\frac{\mathrm{d} \varphi_{i}^{(1)}}{\mathrm{d} x}\right)\left(\varphi_{j}^{(2)}+\frac{\mathrm{d} \varphi_{j}^{(1)}}{\mathrm{d} x}\right)\right] \mathrm{d} x \\
q_{i} & =\int_{x_{a}}^{x_{b}} \varphi_{i}^{(1)} q(x) \mathrm{d} x
\end{aligned}
$$

and $Q_{1}=V_{a}, Q_{2}=M_{a}, Q_{3}=V_{b}$, and $Q_{4}=M_{b}$. Equation (22) has the explicit form,

$$
\left(\frac{2 E I}{\mu h^{3}}\right)\left[\begin{array}{cccc}
6 & -3 h & -6 & -3 h \\
-3 h & 2 h^{2} \lambda & 3 h & h^{2} \xi \\
-6 & 3 h & 6 & 3 h \\
-3 h & h^{2} \xi & 3 h & 2 h^{2} \lambda
\end{array}\right]\left\{\begin{array}{l}
W_{1} \\
\Phi_{1} \\
W_{2} \\
\Phi_{2}
\end{array}\right\}=\left\{\begin{array}{c}
q_{1} \\
q_{2} \\
q_{3} \\
q_{4}
\end{array}\right\}+\left\{\begin{array}{c}
V_{a} \\
M_{a} \\
V_{b} \\
M_{b}
\end{array}\right\} .
$$

This element leads to the exact nodal deflections in static analyses for any distribution of the transverse load $q(x)$ and element-wise constant bending stiffness $E I$ and shear stiffness $G A K_{s}$. Therefore, the element is said to be superconvergent. In the thin beam limit, (25) reduces to the Euler-Bernoulli beam equations, and no additional constraints are implied by the system.

\section{The assumed strain-displacement (ASD) models}

\subsection{General finite element model}

Here we develop the finite element model based on a variational form in which the displacements $\left(w, \phi_{x}\right)$ and strains $\left(\kappa_{x x}, \gamma_{x z}\right)$ are treated as independent field variables. The 
variational statement associated with this mixed formulation is given by the stationarity of the following functional (see Oden \& Reddy 1982, p. 116, equation (4.115)):

$$
\begin{aligned}
R_{\varepsilon}= & \int_{x_{a}}^{x_{b}}\left\{\left[E I\left(\frac{\mathrm{d} \phi_{x}}{\mathrm{~d} x}-\frac{1}{2} \kappa_{x x}\right) \kappa_{x x}\right]\right. \\
& \left.+\left[G A K_{s}\left(\frac{\mathrm{d} w}{\mathrm{~d} x}+\phi_{x}-\frac{1}{2} \gamma_{x z}\right) \gamma_{x z}\right]-q w\right\} \mathrm{d} x \\
& -V_{a} w\left(x_{a}\right)-V_{b} w\left(x_{b}\right)-M_{a} \phi_{x}\left(x_{a}\right)-M_{b} \phi_{x}\left(x_{b}\right),
\end{aligned}
$$

where

$$
\begin{aligned}
V_{a} & =\left[-G A K_{s} \gamma_{x z}\right]_{x_{a}}, & V_{b} & =\left[G A K_{s} \gamma_{x z}\right]_{x_{b}}, \\
M_{a} & =\left[-E I \kappa_{x x}\right]_{x_{a}}, & M_{b} & =\left[E I \kappa_{x x}\right]_{x_{b}} .
\end{aligned}
$$

The first variation of $R_{\varepsilon}$ yields the weak forms

$$
\begin{aligned}
& \int_{x_{a}}^{x_{b}}\left(G A K_{s} \frac{\mathrm{d} \delta w}{\mathrm{~d} x} \gamma_{x z}-\delta w q\right) \mathrm{d} x-V_{a} \delta w\left(x_{a}\right)-V_{b} \delta w\left(x_{b}\right)=0, \\
& \int_{x_{a}}^{x_{b}}\left(E I \frac{\mathrm{d} \delta \phi_{x}}{\mathrm{~d} x} \kappa_{x x}+G A K_{s} \delta \phi_{x} \gamma_{x z}\right) \mathrm{d} x-M_{a} \delta \phi_{x}\left(x_{a}\right)-M_{b} \delta \phi_{x}\left(x_{b}\right)=0, \\
& \int_{x_{a}}^{x_{b}} E I \delta \kappa_{x x}\left(\frac{\mathrm{d} \phi_{x}}{\mathrm{~d} x}-\kappa_{x x}\right) \mathrm{d} x=0, \\
& \int_{x_{a}}^{x_{b}} G A K_{s} \delta \gamma_{x z}\left(\frac{\mathrm{d} w}{\mathrm{~d} x}+\phi_{x}-\gamma_{x z}\right) \mathrm{d} x=0 .
\end{aligned}
$$

Let the variables $\left(w, \phi_{x}, \kappa_{x x}, \gamma_{x z}\right)$ be approximated as

$$
\begin{aligned}
w & \approx \sum_{j=1}^{m} \psi_{j}^{(1)} W_{j}, \quad \phi_{x} \approx \sum_{j=1}^{n} \psi_{j}^{(2)} \Phi_{j}, \\
\kappa_{x x} & \approx \sum_{j=1}^{p} \psi_{j}^{(3)} \mathcal{K}_{j}, \quad \gamma_{x z} \approx \sum_{j=1}^{q} \psi_{j}^{(4)} \Gamma_{j},
\end{aligned}
$$

where $\left(W_{j}, \Phi_{j}, \mathcal{K}_{j}, \Gamma_{j}\right)$ are the nodal values of $\left(w, \phi_{x}, \kappa_{x x}, \gamma_{x z}\right)$ and $\psi_{j}^{(\alpha)}(x)(\alpha=$ $1,2,3,4)$ are the associated interpolation functions whose choice is yet to be made. Substituting (32) into (28)-(31), we obtain the following finite element model:

$$
\left[\begin{array}{cccc}
{[0]} & {[0]} & {[0]} & {[A]} \\
{[0]} & {[0]} & {[B]} & {[C]} \\
{[0]} & {[B]^{T}} & -[D] & {[0]} \\
{[A]^{T}} & {[C]^{T}} & {[0]} & -[G]
\end{array}\right]\left\{\begin{array}{c}
\{W\} \\
\{\Phi\} \\
\{\mathcal{K}\} \\
\{\Gamma\}
\end{array}\right\}=\left\{\begin{array}{c}
\{F\} \\
\{0\} \\
\{0\} \\
\{0\}
\end{array}\right\}+\left\{\begin{array}{c}
\{V\} \\
\{M\} \\
\{0\} \\
\{0\}
\end{array}\right\},
$$

where

$$
\begin{array}{rlrl}
A_{i j} & =\int_{x_{a}}^{x_{b}} G A K_{s} \frac{\mathrm{d} \psi_{i}^{(1)}}{\mathrm{d} x} \psi_{j}^{(4)} \mathrm{d} x, & B_{i j}=\int_{x_{a}}^{x_{b}} E I \frac{\mathrm{d} \psi_{i}^{(2)}}{\mathrm{d} x} \psi_{j}^{(3)} \mathrm{d} x, \\
C_{i j}=\int_{x_{a}}^{x_{b}} G A K_{s} \psi_{i}^{(2)} \psi_{j}^{(4)} \mathrm{d} x, & D_{i j}=\int_{x_{a}}^{x_{b}} E I \psi_{i}^{(3)} \psi_{j}^{(3)} \mathrm{d} x,
\end{array}
$$




$$
\begin{aligned}
G_{i j} & =\int_{x_{a}}^{x_{b}} G A K_{s} \psi_{i}^{(4)} \psi_{j}^{(4)} \mathrm{d} x, \quad F_{i}=\int_{x_{a}}^{x_{b}} q \psi_{i}^{(1)} \mathrm{d} x, \\
V_{1} & =V_{a}, \quad V_{m}=V_{b}, \quad M_{1}=M_{a}, \quad M_{n}=M_{b} .
\end{aligned}
$$

Couple of observations are in order concerning the finite element model in (33). We note that $[A]$ is a vector $\{A\}$ when $\gamma_{x z}$ is approximated as a constant, $\Gamma_{0}$. In addition, the first equation of (33) has the form

$$
G A K_{s}\left\{\begin{array}{c}
-1 \\
0 \\
\vdots \\
0 \\
1
\end{array}\right\} \Gamma_{0}=\left\{\begin{array}{c}
F_{1} \\
F_{2} \\
F_{3} \\
\vdots \\
F_{m}
\end{array}\right\}+\left\{\begin{array}{c}
V_{1} \\
0 \\
\vdots \\
0 \\
V_{m}
\end{array}\right\}
$$

when $w$ is interpolated using quadratic or higher-order polynomials. The nonzero entries correspond to the deflection degrees of freedom at node 1 and node $m$. For linear interpolation of $w$, we have $m=2$ and (35) is alright. However, when $m>2$, (35) implies that $F_{i}=0$ for $i=2, \cdots, m-1$, which, in general, is not true. Thus, either the distributed load is zero or it is converted to generalized point forces at the end nodes through Hermite cubic polynomials. In the latter case, the force components can be added to $V_{a}$ and $V_{b}$ and the moment components to $M_{a}$ and $M_{b}$ at nodes 1 and $m$ respectively.

\subsection{ASD-LLCC element}

For linear (L) interpolation of $\left(w, \phi_{x}\right)$ and constant $(\mathrm{C})$ representation of $\left(\kappa_{x x}, \gamma_{x z}\right)$, and for constant values of $E I$ and $G A K_{s}$, the element equations become ( $m=n=2$ and $p=q=1)$

$$
\begin{aligned}
& G A K_{s}\left\{\begin{array}{c}
-1 \\
1
\end{array}\right\} \Gamma_{0}=\left\{\begin{array}{l}
q_{1}^{(1)} \\
q_{2}^{(2)}
\end{array}\right\}+\left\{\begin{array}{l}
V_{a} \\
V_{b}
\end{array}\right\}, \\
& E I\left\{\begin{array}{c}
-1 \\
1
\end{array}\right\} \mathcal{K}_{0}+G A K_{s} \frac{h}{2}\left\{\begin{array}{l}
1 \\
1
\end{array}\right\} \Gamma_{0}=\left\{\begin{array}{l}
M_{a} \\
M_{b}
\end{array}\right\}, \\
& \{-11\}\left\{\begin{array}{l}
W_{1} \\
W_{2}
\end{array}\right\}+\frac{h}{2}\{11\}\left\{\begin{array}{l}
\Phi_{1} \\
\Phi_{2}
\end{array}\right\}-h \Gamma_{0}=0, \\
& \{-11\}\left\{\begin{array}{l}
\Phi_{1} \\
\Phi_{2}
\end{array}\right\}-h \mathcal{K}_{0}=0 .
\end{aligned}
$$

Solving (38) and (39) for $\mathcal{K}_{0}$ and $\Gamma_{0}$ and substituting into (36) and (37) (i.e., condensing out $\mathcal{K}_{0}$ and $\gamma_{0}$ ), we arrive at the following $4 \times 4$ system of equations,

$$
\begin{aligned}
\frac{G A K_{s}}{4 h} & {\left[\begin{array}{cccc}
4 & -2 h & -4 & -2 h \\
-2 h & h^{2}(1+4 \Omega) & 2 h & h^{2}(1-4 \Omega) \\
-4 & 2 h & 4 & 2 h \\
-2 h & h^{2}(1-4 \Omega) & 2 h & h^{2}(1+4 \Omega)
\end{array}\right]\left\{\begin{array}{l}
W_{1} \\
\Phi_{1} \\
W_{2} \\
\Phi_{2}
\end{array}\right\} } \\
& =\left\{\begin{array}{c}
q_{1}^{(1)} \\
0 \\
q_{2}^{(1)} \\
0
\end{array}\right\}+\left\{\begin{array}{c}
V_{a} \\
M_{a} \\
V_{b} \\
M_{b}
\end{array}\right\},
\end{aligned}
$$


where $\Omega=E I / G A K_{s} h^{2}$. These are exactly the same equations obtained in the displacement formulation with the linear interpolation of $w$ and $\phi_{x}$ and using one-point Gauss quadrature to evaluate the shear stiffnesses, i.e., the reduced integration element (RIE). Thus, the assumed strain-displacement formulation eliminates the need for reduced integration concepts.

\subsection{ASD-HQLC element}

Suppose that the distributed load is represented using

$$
q_{i}^{(h)}=\int_{x_{a}}^{x_{b}} q(x) \varphi_{i}(x) \mathrm{d} x .
$$

A Lagrange or Hermite cubic interpolation of $w$, quadratic interpolation of $\phi_{x}$, linear interpolation of $\kappa_{x x}$, and constant representation of $\gamma_{x z}$ yields the equations

$$
\begin{aligned}
& G A K_{s}\left\{\begin{array}{c}
-1 \\
1
\end{array}\right\} \Gamma_{0}=\left\{\begin{array}{c}
\bar{V}_{a} \\
\bar{V}_{b}
\end{array}\right\}, \\
& \frac{E I}{6}\left[\begin{array}{cc}
-5 & -1 \\
4 & -4 \\
1 & 5
\end{array}\right]\left\{\begin{array}{l}
\mathcal{K}_{1} \\
\mathcal{K}_{2}
\end{array}\right\}+\frac{G A K_{s} h}{6}\left\{\begin{array}{l}
1 \\
4 \\
1
\end{array}\right\} \Gamma_{0}=\left\{\begin{array}{c}
\bar{M}_{a} \\
0 \\
\bar{M}_{b}
\end{array}\right\}, \\
& \frac{E I}{6}\left[\begin{array}{ccc}
-5 & 4 & 1 \\
-1 & -4 & 5
\end{array}\right]\left\{\begin{array}{l}
\Phi_{1} \\
\Phi_{c} \\
\Phi_{2}
\end{array}\right\}-\frac{E I h}{6}\left[\begin{array}{ll}
2 & 1 \\
1 & 2
\end{array}\right]\left\{\begin{array}{l}
\mathcal{K}_{1} \\
\mathcal{K}_{2}
\end{array}\right\}=\left\{\begin{array}{l}
0 \\
0
\end{array}\right\}, \\
& \left\{\begin{array}{ll}
-1 & 1
\end{array}\right\}\left\{\begin{array}{l}
W_{1} \\
W_{2}
\end{array}\right\}+\frac{h}{6}\left\{\begin{array}{lll}
1 & 4 & 1
\end{array}\right\}\left\{\begin{array}{l}
\Phi_{1} \\
\Phi_{c} \\
\Phi_{2}
\end{array}\right\}-h \Gamma_{0}=0,
\end{aligned}
$$

where the end nodes of the element are designated as ' 1 ' and ' 2 ', and the middle node as ' $c$ ', and the interior nodal degrees of freedom associated with $w$ are omitted as they do not contribute to the equations. Solving (44) for $\{\mathcal{K}\}$ and (45) for $\Gamma_{0}$, substituting the result into (42) and (43), and eliminating $\Phi_{c}$, we obtain

$$
\begin{aligned}
& \frac{2 E I}{\mu h^{3}}\left[\begin{array}{cc}
6 & -6 \\
-6 & 6
\end{array}\right]\left\{\begin{array}{l}
W_{1} \\
W_{2}
\end{array}\right\}+\frac{2 E I}{\mu h^{3}}\left[\begin{array}{cc}
-3 h & -3 h \\
3 h & 3 h
\end{array}\right]\left\{\begin{array}{l}
\Phi_{1} \\
\Phi_{2}
\end{array}\right\}=\left\{\begin{array}{l}
\bar{V}_{a} \\
\bar{V}_{b}
\end{array}\right\}, \\
& \frac{2 E I}{\mu h^{3}}\left[\begin{array}{ll}
-3 h & 3 h \\
-3 h & 3 h
\end{array}\right]\left\{\begin{array}{l}
W_{1} \\
W_{2}
\end{array}\right\}+\frac{2 E I}{\mu h^{3}}\left[\begin{array}{cc}
2 h^{2} \lambda & h^{2} \xi \\
h^{2} \xi & 2 h^{2} \lambda
\end{array}\right]\left\{\begin{array}{l}
\Phi_{1} \\
\Phi_{2}
\end{array}\right\}=\left\{\begin{array}{l}
\bar{M}_{a} \\
\bar{M}_{b}
\end{array}\right\} .
\end{aligned}
$$

Adding (46) and (47), we obtain

$$
\left(\frac{2 E I}{\mu h^{3}}\right)\left[\begin{array}{cccc}
6 & -3 h & -6 & -3 h \\
-3 h & 2 h^{2} \lambda & 3 h & h^{2} \xi \\
-6 & 3 h & 6 & 3 h \\
-3 h & h^{2} \xi & 3 h & 2 h^{2} \lambda
\end{array}\right]\left\{\begin{array}{c}
W_{1} \\
\Phi_{1} \\
W_{2} \\
\Phi_{2}
\end{array}\right\}=\left\{\begin{array}{c}
\bar{V}_{a} \\
\bar{M}_{a} \\
\bar{V}_{b} \\
\bar{M}_{b}
\end{array}\right\}
$$

The stiffness matrix is the same as that of the superconvergent element derived by Reddy (1997); however, the load vector is different. It is the same when either the applied load $q$ is element-wise uniform or the load vector is computed using (24) with $\varphi_{i}$ given by (19).

It should be noted that the degree of the polynomial interpolation used for $w$ does not enter the equations presented in all the models discussed in this section. However, the load 
representation implies that $w$ be interpolated with Hermite cubic polynomials or $\varphi_{i}^{(1)}$ of (19). It can be shown that the use of the interdependent interpolations of (19) and (20) for $w$ and $\phi_{x}$ also results in (48).

\section{Two-component form of the Timoshenko beam theory}

\subsection{Theoretical formulation}

The displacement and mixed formulations of the conventional Timoshenko beam theory yield the superconvergent stiffness matrix only when higher-order interpolations of $w$ and $\phi_{x}$ are used. In contrast, the Euler-Bernoulli beam element is superconvergent for the lowest admissible interpolation, namely, the Hermite cubic interpolation. In this section, it is shown that the superconvergent element can be developed with the lowest admissible interpolation of various displacement components. This requires a reformulation of the Timoshenko beam theory in terms of the bending and shear components of the transverse deflection. The two-component form of the transverse deflection was discussed by Anderson (1953), Miklowitz (1953), Huffington (1963), and Krishna Murty (1970) for beams, and Miklowitz (1960), Chow (1971), Bhashyam \& Gallagher (1984), Reddy (1987), Lim et al (1988), and Senthilnathan et al (1988) for plates.

Assume displacement field of the form

$$
u_{1}(x, z)=z\left(-\frac{\mathrm{d} w^{b}}{\mathrm{~d} x}+\beta_{x}\right), \quad u_{2}=0, \quad u_{3}(x, z)=w^{b}(x)+w^{s}(x),
$$

where $w^{b}$ and $w^{s}$ denote the bending and shear components, respectively, of the total transverse deflection $w$ (see Reddy 1999), and $\beta_{x}$ denotes the shear rotation, in addition to the bending rotation, of a transverse normal about the $y$ axis. The strains and the stressstrain relations are given by

$$
\begin{aligned}
& \varepsilon_{x x}=z\left(\frac{\mathrm{d} \beta_{x}}{\mathrm{~d} x}-\frac{\mathrm{d}^{2} w^{b}}{\mathrm{~d} x^{2}}\right), \quad \gamma_{x z}=\left(\beta_{x}+\frac{\mathrm{d} w^{s}}{\mathrm{~d} x}\right), \\
& \sigma_{x x}=E \varepsilon_{x x}, \quad \sigma_{x z}=G \gamma_{x z} .
\end{aligned}
$$

The principle of virtual displacements yields the following Euler-Lagrange equations:

$$
\begin{aligned}
& \delta \beta_{x}:-\frac{\mathrm{d} M}{\mathrm{~d} x}+Q=0, \\
& \delta w^{b}:-\frac{\mathrm{d}^{2} M}{\mathrm{~d} x^{2}}=q, \\
& \delta w^{s}:-\frac{\mathrm{d} Q}{\mathrm{~d} x}=q,
\end{aligned}
$$

where $M(x)$ and $Q(x)$ are bending and shear force resultants,

$$
\begin{aligned}
& M=\int_{A} \sigma_{x x} z \mathrm{~d} A=E I\left(\frac{\mathrm{d} \beta_{x}}{\mathrm{~d} x}-\frac{\mathrm{d}^{2} w^{b}}{\mathrm{~d} x^{2}}\right), \\
& Q=K_{s} \int_{A} \sigma_{x z} \mathrm{~d} A=K_{s} G A\left(\beta_{x}+\frac{\mathrm{d} w^{s}}{\mathrm{~d} x}\right) .
\end{aligned}
$$




\subsection{Finite element model}

The finite element model of the modified Timoshenko beam theory can be developed using the standard steps. The first step is to write the weak forms of the three equations over a typical element. We have

$$
\begin{aligned}
0=\int_{x_{a}}^{x_{b}} & {\left[E I\left(\frac{\mathrm{d} \delta \beta_{x}}{\mathrm{~d} x}-\frac{\mathrm{d}^{2} \delta w^{b}}{\mathrm{~d} x^{2}}\right)\left(\frac{\mathrm{d} \beta_{x}}{\mathrm{~d} x}-\frac{\mathrm{d}^{2} w^{b}}{\mathrm{~d} x^{2}}\right)\right.} \\
& \left.+K_{s} G A\left(\delta \beta_{x}+\frac{\mathrm{d} \delta w^{s}}{\mathrm{~d} x}\right)\left(\beta_{x}+\frac{\mathrm{d} w^{s}}{\mathrm{~d} x}\right)\right] \mathrm{d} x-\int_{x_{a}}^{x_{b}} q\left(\delta w^{b}+\delta w^{s}\right) \mathrm{d} x \\
& -M_{a} \delta \beta_{x}\left(x_{a}\right)-M_{b} \delta \beta_{x}\left(x_{b}\right)-V_{a} \delta w^{s}\left(x_{a}\right)-V_{b} \delta w^{s}\left(x_{b}\right)-Q_{1} \delta w^{b}\left(x_{a}\right) \\
& -Q_{2}\left(-\frac{\mathrm{d} \delta w^{b}}{\mathrm{~d} x}\right)_{x_{a}}-Q_{3} \delta w^{b}\left(x_{b}\right)-Q_{4}\left(-\frac{\mathrm{d} \delta w^{b}}{\mathrm{~d} x}\right)_{x_{b}},
\end{aligned}
$$

where

$$
\begin{aligned}
M_{a} & \equiv-M\left(x_{a}\right)=\left[E I\left(\frac{\mathrm{d} \beta_{x}}{\mathrm{~d} x}-\frac{\mathrm{d}^{2} w^{b}}{\mathrm{~d} x^{2}}\right)\right]_{x_{a}}, \\
M_{b} & \equiv M\left(x_{b}\right)=\left[-E I\left(\frac{\mathrm{d} \beta_{x}}{\mathrm{~d} x}-\frac{\mathrm{d}^{2} w^{b}}{\mathrm{~d} x^{2}}\right)\right]_{x_{b}}, \\
V_{a} & \equiv-Q\left(x_{a}\right)=\left[K_{s} G A\left(\beta_{x}+\frac{\mathrm{d} w^{s}}{\mathrm{~d} x}\right)\right]_{x_{a}}, \\
V_{b} & \equiv Q\left(x_{b}\right)=\left[-K_{s} G A\left(\beta_{x}+\frac{\mathrm{d} w^{s}}{\mathrm{~d} x}\right)\right]_{x_{b}} \\
Q_{1} & \equiv-\left(\frac{\mathrm{d} M}{\mathrm{~d} x}\right)_{x_{a}}=-Q\left(x_{a}\right)=V_{a} \\
Q_{3} & \equiv\left(\frac{\mathrm{d} M}{\mathrm{~d} x}\right)_{x_{b}}=Q\left(x_{b}\right)=V_{b}, \\
Q_{2} & \equiv-M\left(x_{a}\right)=M_{a}, \quad Q_{4} \equiv M\left(x_{b}\right)=M_{b} .
\end{aligned}
$$

From the weak form (57), it is clear that $\beta_{x}$ and $w^{s}$ can be interpolated using the Lagrange interpolation and $w^{b}$ using Hermite interpolation. The lowest admissible functions are linear for $\beta_{x}$ and $w^{s}$ and cubic for $w^{b}$. However, the condition that the shear force be element-wise constant for element-wise constant values of $E I$ in turn requires that $w^{s}$ be quadratic.

Let $\left(\beta_{x}, w^{b}, w^{s}\right)$ be interpolated as

$$
\begin{aligned}
\beta_{x}(x) & \approx \sum_{i=1}^{m} \mathcal{B}_{i} \psi_{i}^{(1)}(x), \quad w^{s}(x) \approx \sum_{i=1}^{n} W_{i}^{s} \psi_{i}^{(2)}(x), \\
w^{b}(x) & \approx \sum_{i=1}^{p} W_{i}^{b} \varphi_{i}(x),
\end{aligned}
$$


where $\mathcal{B}_{i}, W_{i}^{s}$ and $W_{i}^{b}$ denote the nodal values of $\beta_{x}, w^{s}$ and $w^{b}$, respectively, $\psi_{i}^{(1)}$ and $\psi_{i}^{(2)}$ are linear and quadratic interpolation functions, respectively, and $\varphi_{i}$ are the Hermite cubic interpolation functions ( $m=2, n=3, p=4$ ). Substituting the interpolations (59) into the weak form (57), we obtain the following finite element model:

or simply

$$
\left[\begin{array}{ccc}
{[A]} & {[B]} & {[C]} \\
{[B]^{T}} & {[D]} & {[0]} \\
{[C]^{T}} & {[0]} & {[G]}
\end{array}\right]\left\{\begin{array}{c}
\{\mathcal{B}\} \\
\left\{W^{b}\right\} \\
\left\{W^{s}\right\}
\end{array}\right\}=\left\{\begin{array}{c}
\{0\} \\
\left\{F^{b}\right\} \\
\left\{F^{s}\right\}
\end{array}\right\}+\left\{\begin{array}{c}
\{M\} \\
\{Q\} \\
\{V\}
\end{array}\right\},
$$

$$
\left[K^{R}\right]\{\Delta\}=\left\{F^{R}\right\}
$$

where the stiffness matrix $\left[K^{R}\right]$ is of the order $9 \times 9$.

The coefficients of various matrices and vectors in $(60)$ are defined by

$$
\begin{aligned}
A_{i j} & =\int_{x_{a}}^{x_{b}}\left(E I \frac{\mathrm{d} \psi_{i}^{(1)}}{\mathrm{d} x} \frac{\mathrm{d} \psi_{j}^{(1)}}{\mathrm{d} x}+K_{s} G A \psi_{i}^{(1)} \psi_{j}^{(1)}\right) \mathrm{d} x, \\
B_{i j} & =-\int_{x_{a}}^{x_{b}} E I \frac{\mathrm{d} \psi_{i}^{(1)}}{\mathrm{d} x} \frac{\mathrm{d}^{2} \varphi_{j}}{\mathrm{~d} x^{2}} \mathrm{~d} x, \\
C_{i j} & =\int_{x_{a}}^{x_{b}} K_{s} G A \psi_{i}^{(1)} \frac{\mathrm{d} \psi_{j}^{(2)}}{\mathrm{d} x} \mathrm{~d} x, \\
D_{i j} & =\int_{x_{a}}^{x_{b}} E I \frac{\mathrm{d}^{2} \varphi_{i}}{\mathrm{~d} x^{2}} \frac{\mathrm{d}^{2} \varphi_{j}}{\mathrm{~d} x^{2}} \mathrm{~d} x, \\
G_{i j} & =\int_{x_{a}}^{x_{b}} K_{s} G A \frac{\mathrm{d} \psi_{i}^{(2)}}{\mathrm{d} x} \frac{\mathrm{d} \psi_{j}^{(2)}}{\mathrm{d} x} \mathrm{~d} x, \\
F_{i}^{b} & =\int_{x_{a}}^{x_{b}} q \varphi_{i} \mathrm{~d} x, \quad F_{i}^{s}=\int_{x_{a}}^{x_{b}} q \psi_{i}^{(2)} \mathrm{d} x .
\end{aligned}
$$

The element equations (60) are not suitable for practical use. The reason is that we only know the total displacement $w=w^{b}+w^{s}$ and not its bending and shear parts separately. This is also true about the total rotation $\left(\phi_{x}=-w_{, x}^{b}+\beta_{x}\right)$. Hence, it is necessary to recast the element equations (60) in terms of the physical nodal variables.

\subsection{Reduction of equations}

Here we select specific interpolation functions and evaluate the element matrices. For the choice of linear interpolation functions for $\psi_{i}^{(1)}$, quadratic interpolation functions for $\psi_{i}^{(2)}$, and Hermite cubic interpolations functions for $\varphi_{i}$ (the minimum polynomials required by the weak form), we obtain (see figure 2)

$$
\begin{aligned}
& \left(\frac{E I}{h}\left[\begin{array}{cc}
1 & -1 \\
-1 & 1
\end{array}\right]+\frac{K_{s} G A h}{6}\left[\begin{array}{ll}
2 & 1 \\
1 & 2
\end{array}\right]\right)\left\{\begin{array}{l}
\mathcal{B}_{1} \\
\mathcal{B}_{2}
\end{array}\right\}+\frac{E I}{h}\left[\begin{array}{cc}
1 & -1 \\
-1 & 1
\end{array}\right]\left\{\begin{array}{l}
W_{2}^{b} \\
W_{4}^{b}
\end{array}\right\} \\
& +\frac{K_{s} G A}{6}\left[\begin{array}{ccc}
-5 & 4 & 1 \\
-1 & -4 & 5
\end{array}\right]\left\{\begin{array}{l}
W_{1}^{s} \\
W_{c}^{s} \\
W_{2}^{s}
\end{array}\right\}=\left\{\begin{array}{l}
M_{a} \\
M_{b}
\end{array}\right\}
\end{aligned}
$$




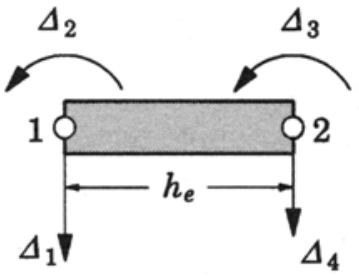

Primary deg. of freedom

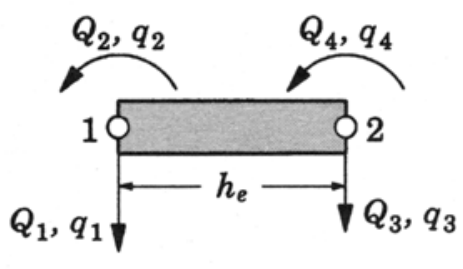

Secondary deg. of freedom

(a)

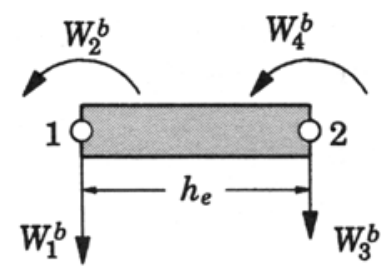

Primary variables associated with $w^{b}$

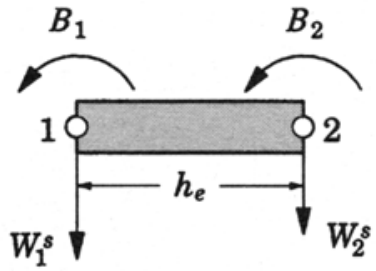

Primary variables associated with $w^{s}$ and $\beta$

(b)

Figure 2. Force and displacement degrees of freedom. (a) Total degrees of freedom. (b) Displacement degrees of freedom associated with $w^{b}, w^{s}$, and $\beta$.

$$
\begin{aligned}
& \frac{12 E I}{h^{3}}\left[\begin{array}{cc}
1 & -1 \\
-1 & 1
\end{array}\right]\left\{\begin{array}{l}
W_{1}^{b} \\
W_{3}^{b}
\end{array}\right\}+\frac{6 E I}{h^{2}}\left[\begin{array}{cc}
-1 & -1 \\
1 & 1
\end{array}\right]\left\{\begin{array}{l}
W_{2}^{b} \\
W_{4}^{b}
\end{array}\right\} \\
& =\left\{\begin{array}{l}
Q_{1} \\
Q_{3}
\end{array}\right\}+\left\{\begin{array}{l}
q_{1}^{(h)} \\
q_{3}^{(h)}
\end{array}\right\} \\
& \frac{6 E I}{h^{2}}\left[\begin{array}{ll}
-1 & 1 \\
-1 & 1
\end{array}\right]\left\{\begin{array}{l}
W_{1}^{b} \\
W_{3}^{b}
\end{array}\right\}+\frac{2 E I}{h}\left[\begin{array}{ll}
2 & 1 \\
1 & 2
\end{array}\right]\left\{\begin{array}{l}
W_{2}^{b} \\
W_{4}^{b}
\end{array}\right\} \\
& -\frac{E I}{h}\left[\begin{array}{cc}
1 & -1 \\
-1 & 1
\end{array}\right]\left\{\begin{array}{l}
\mathcal{B}_{1} \\
\mathcal{B}_{2}
\end{array}\right\}=\left\{\begin{array}{l}
Q_{2} \\
Q_{4}
\end{array}\right\}+\left\{\begin{array}{l}
q_{2}^{(h)} \\
q_{4}^{(h)}
\end{array}\right\} \\
& \frac{K_{s} G A}{6}\left[\begin{array}{cc}
-5 & -1 \\
4 & -4 \\
1 & 5
\end{array}\right]\left\{\begin{array}{l}
\mathcal{B}_{1} \\
\mathcal{B}_{2}
\end{array}\right\}+\frac{K_{s} G A}{3 h}\left[\begin{array}{ccc}
7 & -8 & 1 \\
-8 & 16 & -8 \\
1 & -8 & 7
\end{array}\right]\left\{\begin{array}{l}
W_{1}^{s} \\
W_{c}^{s} \\
W_{2}^{s}
\end{array}\right\} \\
& =\left\{\begin{array}{l}
V_{a} \\
\hat{Q}_{c} \\
V_{b}
\end{array}\right\}+\left\{\begin{array}{l}
q_{1}^{(2)} \\
q_{c}^{(2)} \\
q_{2}^{(2)}
\end{array}\right\}
\end{aligned}
$$

where

$$
F_{i}^{b}=q_{i}^{(h)}(i=1,2,3,4), \quad F_{i}^{s}=q_{i}^{(2)}(i=1,2, \mathrm{c})
$$


$W_{c}^{s}$ denotes the value of $w^{s}$ and $\hat{Q}_{c}$. is the specified transverse load at the centre node of the element. Note that the finite element equations associated with the second equation in $(60)$ is split into a pair of equations for convenience.

As noted earlier, it is necessary to combine the two components of the transverse deflection as well as the rotation into total deflection and rotation. This amounts to rewriting the algebraic equations $(60)$ to obtain a model solely in terms of the total deflection $w=w^{b}+w^{s}$ and rotation $\phi_{x}=-w_{, x}^{b}+\beta_{x}$ at the element nodes. First we condense out $W_{c}^{s}$ using the second equation of (66). We have

$$
W_{c}=\frac{6 h}{32 G A K_{s}} \hat{F}_{c}+\left(\frac{W_{1}+W_{2}}{2}\right)+h\left(\frac{\Phi_{2}-\Phi_{1}}{8}\right),
$$

where $\hat{F}_{c}=q_{c}^{(2)}+\hat{Q}_{c}$. Substituting (68) into (63) and (66), we obtain

$$
\begin{gathered}
12 \Omega \frac{E I}{h}\left[\begin{array}{cc}
1 & -1 \\
-1 & 1
\end{array}\right]\left\{\begin{array}{l}
\Delta_{2} \\
\Delta_{4}
\end{array}\right\}+\frac{3 E I}{h}\left[\begin{array}{ll}
1 & 1 \\
1 & 1
\end{array}\right]\left\{\begin{array}{l}
\mathcal{B}_{1} \\
\mathcal{B}_{2}
\end{array}\right\} \\
+\frac{6 E I}{h^{2}}\left[\begin{array}{cc}
-1 & 1 \\
-1 & 1
\end{array}\right]\left\{\begin{array}{l}
W_{1}^{s} \\
W_{2}^{s}
\end{array}\right\}=12 \Omega\left(\left\{\begin{array}{l}
M_{a} \\
M_{b}
\end{array}\right\}-\frac{h \hat{F}_{c}^{s}}{8}\left\{\begin{array}{c}
1 \\
-1
\end{array}\right\}\right), \\
\frac{6 E I}{h^{2}}\left[\begin{array}{cc}
-1 & -1 \\
1 & 1
\end{array}\right]\left\{\begin{array}{l}
\mathcal{B}_{1} \\
\mathcal{B}_{2}
\end{array}\right\}+\frac{12 E I}{h^{3}}\left[\begin{array}{cc}
1 & -1 \\
-1 & 1
\end{array}\right]\left\{\begin{array}{l}
W_{1}^{s} \\
W_{2}^{s}
\end{array}\right\} \\
=12 \Omega\left(\left\{\begin{array}{l}
V_{a} \\
V_{b}
\end{array}\right\}+\left\{\begin{array}{l}
q_{1}^{(2)}+\frac{1}{2} q_{c}^{(2)} \\
q_{2}^{(2)}+\frac{1}{2} q_{c}^{(2)}
\end{array}\right\}\right),
\end{gathered}
$$

where $\Delta_{i}$ denote the total generalized displacements,

$$
\begin{array}{ll}
\Delta_{1} \equiv w\left(x_{a}\right)=W_{1}^{b}+W_{1}^{s}, & \Delta_{2} \equiv\left(-\frac{\mathrm{d} w^{b}}{\mathrm{~d} x}+\beta_{x}\right)_{x_{a}}=W_{2}^{b}+\mathcal{B}_{1}, \\
\Delta_{3} \equiv w\left(x_{b}\right)=W_{3}^{b}+W_{2}^{s}, & \Delta_{4} \equiv\left(-\frac{\mathrm{d} w^{b}}{\mathrm{~d} x}+\beta_{x}\right)_{x_{b}}=W_{4}^{b}+\mathcal{B}_{2} .
\end{array}
$$

Adding (64) to (70) and (65) to (69), we find

$$
\begin{gathered}
\left(\frac{2 E I}{h^{3}}\right)\left[\begin{array}{cc}
-6 & 6 \\
6 & -6
\end{array}\right]\left\{\begin{array}{l}
\Delta_{1} \\
\Delta_{3}
\end{array}\right\}+\frac{2 E I}{h^{3}}\left[\begin{array}{cc}
-3 h & -3 h \\
3 h & 3 h
\end{array}\right]\left\{\begin{array}{l}
\Delta_{2} \\
\Delta_{4}
\end{array}\right\} \\
=(1+12 \Omega)\left\{\begin{array}{l}
Q_{1} \\
Q_{3}
\end{array}\right\}+\left\{\begin{array}{c}
q_{1}^{(h)} \\
q_{3}^{(h)}
\end{array}\right\}+12 \Omega\left\{\begin{array}{c}
q_{1}^{(2)}+\frac{1}{2} q_{c}^{(2)} \\
q_{2}^{(2)}+\frac{1}{2} q_{c}^{(2)}
\end{array}\right\}, \\
\frac{2 E I}{h^{3}}\left[\begin{array}{cc}
3 h & 3 h \\
-3 h & 3 h
\end{array}\right]\left\{\begin{array}{l}
\Delta_{1} \\
\Delta_{3}
\end{array}\right\}+\frac{2 E I}{h^{3}}\left[\begin{array}{cc}
2 h^{2} \lambda & h^{2} \xi \\
h^{2} \xi & 2 h^{2} \lambda
\end{array}\right]\left\{\begin{array}{c}
\Delta_{2} \\
\Delta_{4}
\end{array}\right\} \\
=(1+12 \Omega)\left\{\begin{array}{l}
Q_{2} \\
Q_{4}
\end{array}\right\}+\left\{\begin{array}{c}
q_{2}^{(h)} \\
q_{4}^{(h)}
\end{array}\right\}+12 \Omega\left\{\begin{array}{c}
-\frac{h}{8} q_{c}^{(2)} \\
\frac{h}{8} q_{c}^{(2)}
\end{array}\right\} .
\end{gathered}
$$


Now combining (72) and (73), we arrive at

$$
\begin{aligned}
\frac{2 E I}{\mu h^{3}}\left[\begin{array}{cccc}
6 & -3 h & -6 & -3 h \\
-3 h & 2 h^{2} \lambda & 3 h & h^{2} \xi \\
-6 & 3 h & 6 & 3 h \\
-3 h & h^{2} \xi & 3 h & 2 h^{2} \lambda
\end{array}\right]\left\{\begin{array}{l}
\Delta_{1} \\
\Delta_{2} \\
\Delta_{3} \\
\Delta_{4}
\end{array}\right\}=\left\{\begin{array}{l}
Q_{1} \\
Q_{2} \\
Q_{3} \\
Q_{4}
\end{array}\right\} \\
+\frac{1}{\mu}\left\{\begin{array}{c}
q_{1}^{(h)} \\
q_{2}^{(h)} \\
q_{3}^{(h)} \\
q_{4}^{(h)}
\end{array}\right\}+12 \frac{\Omega}{\mu}\left\{\begin{array}{c}
q_{1}^{(2)}+\frac{1}{2} q_{c}^{(2)} \\
-\frac{h}{8} q_{c}^{(2)} \\
q_{2}^{(2)}+\frac{1}{2} q_{c}^{(2)} \\
\frac{h}{8} q_{c}^{(2)}
\end{array}\right\},
\end{aligned}
$$

where

$$
\lambda=1+3 \Omega, \quad \xi=1-6 \Omega, \quad \mu=1+12 \Omega, \quad \Omega=\frac{E I}{K_{s} G A h^{2}} .
$$

Equation (74) is the same as (25) (see Reddy 1999).

\section{Finite element models for dynamic analysis}

\subsection{Weak forms and finite element models}

For the dynamic case, the weak forms in (5), (28) and (29), and (57) (which correspond to the displacement and mixed finite element models of the conventional Timoshenko beam theory and the displacement model of the modified Timoshenko beam theory) must be modified to read

$$
\begin{aligned}
& 0=\int_{x_{a}}^{x_{b}}\left[E I \frac{\partial \delta \phi_{x}}{\partial x} \frac{\partial \phi_{x}}{\partial x}+G A K_{s}\left(\delta \phi_{x}+\frac{\partial \delta w}{\partial x}\right)\left(\phi_{x}+\frac{\partial w}{\partial x}\right)\right. \\
& \left.-q \delta w+I_{0} \delta w \frac{\partial^{2} w}{\partial t^{2}}+I_{2} \delta \phi_{x} \frac{\partial^{2} \phi_{x}}{\partial t^{2}}\right] \mathrm{d} x-V_{a} \delta w\left(x_{a}, t\right) \\
& -V_{b} \delta w\left(x_{b}, t\right)-M_{a} \delta \phi_{x}\left(x_{a}, t\right)-M_{b} \delta \phi_{x}\left(x_{b}, t\right), \\
& 0=\int_{x_{a}}^{x_{b}}\left(G A K_{s} \frac{\partial \delta w}{\partial x} \gamma_{x z}+I_{0} \delta w \frac{\partial^{2} w}{\partial t^{2}}-\delta w q\right) \mathrm{d} x \\
& -V_{a} \delta w\left(x_{a}, t\right)-V_{b} \delta w\left(x_{b}, t\right), \\
& 0=\int_{x_{a}}^{x_{b}}\left(E I \frac{\partial \delta \phi_{x}}{\partial x} \kappa_{x x}+G A K_{s} \delta \phi_{x} \gamma_{x z}+I_{2} \delta \phi_{x} \frac{\partial^{2} \phi_{x}}{\partial t^{2}}\right) \mathrm{d} x \\
& -M_{a} \delta \phi_{x}\left(x_{a}, t\right)-M_{b} \delta \phi_{x}\left(x_{b}, t\right), \\
& 0=\int_{x_{a}}^{x_{b}}\left[E I\left(\frac{\partial \delta \beta_{x}}{\partial x}-\frac{\partial^{2} \delta w^{b}}{\partial x^{2}}\right)\left(\frac{\partial \beta_{x}}{\partial x}-\frac{\partial^{2} w^{b}}{\partial x^{2}}\right)\right. \\
& +K_{s} G A\left(\delta \beta_{x}+\frac{\partial \delta w^{s}}{\partial x}\right)\left(\beta_{x}+\frac{\partial w^{s}}{\partial x}\right)-q\left(\delta w^{b}+\delta w^{s}\right)
\end{aligned}
$$




$$
\begin{aligned}
& \left.+I_{2}\left(-\frac{\partial \ddot{w}^{b}}{\partial x}+\ddot{\beta}_{x}\right)\left(-\frac{\partial \delta w^{b}}{\partial x}+\delta \beta_{x}\right)+I_{0}\left(\ddot{w}^{b}+\ddot{w}^{s}\right)\left(\delta w^{b}+\delta w^{s}\right)\right] \mathrm{d} x \\
& -M_{a} \delta \beta_{x}\left(x_{a}, t\right)-M_{b} \delta \beta_{x}\left(x_{b}, t\right)-V_{a} \delta w^{s}\left(x_{a}, t\right)-V_{b} \delta w^{s}\left(x_{b}, t\right) \\
& -Q_{1} \delta w^{b}\left(x_{a}, t\right)-Q_{2}\left(-\frac{\partial \delta w^{b}}{\partial x}\right)_{x_{a}} \\
& -Q_{3} \delta w^{b}\left(x_{b}, t\right)-Q_{4}\left(-\frac{\partial \delta w^{b}}{\partial x}\right)_{x_{b}}
\end{aligned}
$$

respectively, where

$$
I_{0}=\int_{A} \rho \mathrm{d} A=\rho A, \quad I_{2}=\int_{A} \rho z^{2} d A=\frac{\rho A h^{2}}{12},
$$

$\rho$ being the mass density of the material.

For the dynamic case, the finite element models in (8), (33), and (60) take the following forms.

Reduced integration element (RIE):

$$
\left[\begin{array}{cc}
{\left[M^{11}\right]} & {[0]} \\
{[0]} & {\left[M^{22}\right]}
\end{array}\right]\left\{\begin{array}{c}
\{\ddot{W}\} \\
\{\ddot{\Phi}\}
\end{array}\right\}+\left[\begin{array}{cc}
{\left[K^{11}\right]} & {\left[K^{12}\right]} \\
{\left[K^{12}\right]^{\mathrm{T}}} & {\left[K^{22}\right]}
\end{array}\right]\left\{\begin{array}{c}
\{W\} \\
\{\Phi\}
\end{array}\right\}=\left\{\begin{array}{l}
\left\{F^{1}\right\} \\
\left\{F^{2}\right\}
\end{array}\right\}
$$

Interdependent interpolation element (IIE):

$$
[M]\{\ddot{\Delta}\}+[K]\{\Delta\}=\{q\}+\{Q\}
$$

Assumed strain-displacement model (ASD):

$$
\begin{gathered}
{\left[\begin{array}{cccc}
{\left[M^{11}\right]} & {[0]} & {[0]} & {[0]} \\
{[0]} & {\left[M^{22}\right]} & {[0]} & {[0]} \\
{[0]} & {[0]} & {[0]} & {[0]} \\
{[0]} & {[0]} & {[0]} & {[0]}
\end{array}\right]\left\{\begin{array}{l}
\{\ddot{W}\} \\
\{\ddot{\Phi}\} \\
\{\ddot{\mathcal{K}}\} \\
\{\ddot{\Gamma}\}
\end{array}\right\}+\left[\begin{array}{cccc}
{[0]} & {[0]} & {[0]} & {[A]} \\
{[0]} & {[0]} & {[B]} & {[C]} \\
{[0]} & {[B]^{T}} & -[D] & {[0]} \\
{[A]^{T}} & {[C]^{T}} & {[0]} & -[G]
\end{array}\right]} \\
\times\left\{\begin{array}{l}
\{W\} \\
\{\Phi\} \\
\{\mathcal{K}\} \\
\{\Gamma\}
\end{array}\right\}=\left\{\begin{array}{l}
\{F\} \\
\{0\} \\
\{0\} \\
\{0\}
\end{array}\right\}+\left\{\begin{array}{l}
\{V\} \\
\{M\} \\
\{0\} \\
\{0\}
\end{array}\right\} .
\end{gathered}
$$

Two-component theory displacement finite element model:

$$
\begin{aligned}
& {\left[\begin{array}{ccc}
{\left[M^{1}\right]} & -\left[M^{2}\right] & {[0]} \\
-\left[M^{2}\right]^{\mathrm{T}} & {\left[M^{3}\right]} & {\left[M^{4}\right]} \\
{[0]} & {\left[M^{4}\right]^{\mathrm{T}}} & {\left[M^{5}\right]}
\end{array}\right]\left\{\begin{array}{c}
\{\ddot{\mathcal{B}}\} \\
\left\{\ddot{W}^{b}\right\} \\
\left\{\ddot{W}^{s}\right\}
\end{array}\right\}+\left[\begin{array}{ccc}
{[A]} & {[B]} & {[C]} \\
{[B]^{T}} & {[D]} & {[0]} \\
{[C]^{T}} & {[0]} & {[G]}
\end{array}\right]\left\{\begin{array}{c}
\{\mathcal{B}\} \\
\left\{W^{b}\right\} \\
\left\{W^{s}\right\}
\end{array}\right\}} \\
& =\left\{\begin{array}{c}
\{0\} \\
\left\{F^{b}\right\} \\
\left\{F^{s}\right\}
\end{array}\right\}+\left\{\begin{array}{l}
\{M\} \\
\{Q\} \\
\{V\}
\end{array}\right\}
\end{aligned}
$$


where

$$
\begin{aligned}
M_{i j} & =\int_{x_{a}}^{x_{b}}\left(I_{0} \varphi_{i}^{(1)} \varphi_{j}^{(1)}+I_{2} \varphi_{i}^{(2)} \varphi_{j}^{(2)}\right) \mathrm{d} x \\
M_{i j}^{11} & =\int_{x_{a}}^{x_{b}} I_{0} \psi_{i}^{(1)} \psi_{j}^{(1)} \mathrm{d} x, \quad M_{i j}^{22}=\int_{x_{a}}^{x_{b}} I_{2} \psi_{i}^{(2)} \psi_{j}^{(2)} \mathrm{d} x \\
M_{i j}^{1} & =\int_{x_{a}}^{x_{b}} I_{2} \psi_{i}^{(1)} \psi_{j}^{(1)} \mathrm{d} x, \quad M_{i j}^{2}=\int_{x_{a}}^{x_{b}} I_{2} \psi_{i}^{(1)} \frac{\mathrm{d} \varphi_{j}}{\mathrm{~d} x} \mathrm{~d} x \\
M_{i j}^{3} & =\int_{x_{a}}^{x_{b}}\left(I_{0} \varphi_{i} \varphi_{j}+I_{2} \frac{\mathrm{d} \varphi_{i}}{\mathrm{~d} x} \frac{\mathrm{d} \varphi_{j}}{\mathrm{~d} x}\right) \mathrm{d} x, \\
M_{i j}^{4} & =\int_{x_{a}}^{x_{b}} I_{0} \varphi_{i} \psi_{j}^{(2)} \mathrm{d} x, \quad M_{i j}^{5}=\int_{x_{a}}^{x_{b}} I_{0} \psi_{i}^{(2)} \psi_{j}^{(2)} \mathrm{d} x
\end{aligned}
$$

See the previous sections for the definition of the stiffness coefficients and displacement and load vectors. The specific forms of the mass matrices are given in the next section.

\subsection{Mass matrices}

Because of the presence of the second time-derivative terms $\ddot{W}$ and $\ddot{\Phi}$, it is not possible to algebraically manipulate the equations, as was done in the static case for CIE, ASDHQLC, and finite element model based on the two-component form of the Timoshenko beam theory. Recall that for RIE (linear or quadratic), IIE, and ASD-LLCC, no algebraic manipulations were necessary. Therefore, these elements are directly applicable to the dynamic analysis. For the finite element model based on the two-component form of the Timoshenko beam theory, one may select a mass matrix to go with the superconvergent (SCE) stiffness matrix for the dynamic analysis. Additional discussion of this point will follow. The explicit forms of the finite element equations for the three models, RIE, IIE, and SCE are summarized below.

Reduced integration element (RIE): For linear interpolation of $w$ and $\phi_{x}$, the finite element equations are given by

$$
\begin{aligned}
\frac{h}{6}\left[\begin{array}{cccc}
2 I_{0} & 0 & I_{0} & 0 \\
0 & 2 I_{2} & 0 & I_{2} \\
I_{0} & 0 & 2 I_{0} & 0 \\
0 & I_{2} & 0 & 2 I_{2}
\end{array}\right]\left\{\begin{array}{l}
\ddot{W}_{1} \\
\ddot{\Phi}_{1} \\
\ddot{W}_{2} \\
\ddot{\Phi}_{2}
\end{array}\right\}+\frac{2 E I}{\mu_{0} h^{3}} \\
\times\left[\begin{array}{cccc}
6 & -3 h & -6 & -3 h \\
-3 h & h^{2}(1.5+6 \Omega) & 3 h & h^{2}(1.5-6 \Omega) \\
-6 & 3 h & 6 & 3 h \\
-3 h & h^{2}(1.5-6 \Omega) & 3 h & h^{2}(1.5+6 \Omega)
\end{array}\right]\left\{\begin{array}{c}
W_{1} \\
\Phi_{1} \\
W_{2} \\
\Phi_{2}
\end{array}\right\} \\
=\left\{\begin{array}{c}
q_{1}^{(1)} \\
0 \\
q_{2}^{(1)} \\
0
\end{array}\right\}+\left\{\begin{array}{c}
V_{a} \\
M_{a} \\
V_{b} \\
M_{b}
\end{array}\right\}
\end{aligned}
$$


For quadratic interpolation of both $w$ and $\phi_{x}$, the element matrices are of the order $6 \times 6$ for pure bending case.

Interdependent interpolation element (IIE): For this case, the stiffness matrix and load vector are given in (25) [and the same as in (74)]. The mass matrix [M] of (85) consists of several parts, as given below.

$$
\begin{aligned}
& {[M]=\frac{I_{0} h}{420 \mu^{2}}\left[\begin{array}{cccc}
156 & -22 h & 54 & 13 h \\
-22 h & 4 h^{2} & -13 h & -3 h^{2} \\
54 & -13 h & 156 & 22 h \\
13 h & -3 h^{2} & 22 h & 4 h^{2}
\end{array}\right]+\frac{I_{2}}{30 h \mu^{2}}\left[\begin{array}{cccc}
36 & -3 h & -36 & -3 h \\
-3 h & 4 h^{2} & 3 h & -h^{2} \\
-36 & -3 h & 36 & 3 h \\
-3 h & -h^{2} & 3 h & 4 h^{2}
\end{array}\right]} \\
& +\Omega\left(\frac{I_{0} h}{10 \mu^{2}}\left[\begin{array}{cccc}
84 & -11 h & 36 & 9 h \\
-11 h & 2 h^{2} & -9 h & -2 h^{2} \\
36 & -9 h & 84 & 11 h \\
9 h & -2 h^{2} & 11 h & 2 h^{2}
\end{array}\right]+\frac{I_{2}}{\mu^{2}}\left[\begin{array}{cccc}
0 & 6 & 0 & 6 \\
6 & 2 h & -6 & -2 h \\
0 & -6 & 0 & -6 \\
6 & -2 h & -6 & 2 h
\end{array}\right]\right) \\
& +\Omega^{2}\left(\frac{I_{0} h}{5 \mu^{2}}\left[\begin{array}{cccc}
240 & -30 h & 120 & 30 h \\
-30 h & 6 h^{2} & -30 h & -6 h^{2} \\
120 & -30 h & 240 & 30 h \\
30 h & -6 h^{2} & 30 h & 6 h^{2}
\end{array}\right]+\frac{24 I_{2} h}{\mu^{2}}\left[\begin{array}{llll}
0 & 0 & 0 & 0 \\
0 & 2 & 0 & 1 \\
0 & 0 & 0 & 0 \\
0 & 1 & 0 & 2
\end{array}\right]\right) \text {. }
\end{aligned}
$$

Finite element model with superconvergent stiffness matrix (SCE): Although the superconvergent form of the stiffness matrix can be derived using various approaches, only the interdependent interpolation element formulation is readily extendable to the dynamic case (see (82) and (88)). The other formulations do not permit the algebraic manipulations with the mass terms in place. Hence, one may choose a mass matrix to go with (48) and (74). There are several choices: (i) use the same mass matrix as in (88), (ii) use the mass matrix of the Euler-Bernoulli beam element, or (iii) use the mass matrix of the IIE element with $\Omega=0$ (hence, $\mu=1$ ). The first choice reduces the formulation to IIE. The second and third choices are the same because of the relationship between $\varphi_{i}^{(1)}, \varphi_{i}^{(2)}$, and $\varphi_{i}$ [see the comment after (21)]. Thus, for the dynamic case, the finite element model in (74) takes the form

$$
\begin{aligned}
& \left(\frac{I_{0} h}{420}\left[\begin{array}{cccc}
156 & -22 h & 54 & 13 h \\
-22 h & 4 h^{2} & -13 h & -3 h^{2} \\
54 & -13 h & 156 & 22 h \\
13 h & -3 h^{2} & 22 h & 4 h^{2}
\end{array}\right]\right. \\
& \left.+\frac{I_{2}}{30 h}\left[\begin{array}{cccc}
36 & -3 h & -36 & -3 h \\
-3 h & 4 h^{2} & 3 h & -h^{2} \\
-36 & -3 h & 36 & 3 h \\
-3 h & -h^{2} & 3 h & 4 h^{2}
\end{array}\right]\right)\left\{\begin{array}{l}
\ddot{\Delta}_{1} \\
\ddot{\Delta}_{2} \\
\ddot{\Delta}_{3} \\
\ddot{\Delta}_{4}
\end{array}\right\} \\
& +\frac{2 E I}{\mu h^{3}}\left[\begin{array}{cccc}
6 & -3 h & -6 & -3 h \\
-3 h & 2 h^{2} \lambda & 3 h & h^{2} \xi \\
-6 & 3 h & 6 & 3 h \\
-3 h & h^{2} \xi & 3 h & 2 h^{2} \lambda
\end{array}\right]\left\{\begin{array}{l}
\Delta_{1} \\
\Delta_{2} \\
\Delta_{3} \\
\Delta_{4}
\end{array}\right\}=\left\{\begin{array}{l}
Q_{1} \\
Q_{2} \\
Q_{3} \\
Q_{4}
\end{array}\right\}+\left\{\begin{array}{l}
q_{1} \\
q_{2} \\
q_{3} \\
q_{4}
\end{array}\right\}
\end{aligned}
$$


where

$$
\left\{\begin{array}{l}
q_{1} \\
q_{2} \\
q_{3} \\
q_{4}
\end{array}\right\}=\frac{1}{\mu}\left(\left\{\begin{array}{c}
q_{1}^{(h)} \\
q_{2}^{(h)} \\
q_{3}^{(h)} \\
q_{4}^{(h)}
\end{array}\right\}+12 \Omega\left\{\begin{array}{c}
q_{1}^{(2)}+\frac{1}{2} q_{c}^{(2)} \\
-\frac{h}{8} q_{c}^{(2)} \\
q_{2}^{(2)}+\frac{1}{2} q_{c}^{(2)} \\
\frac{h}{8} q_{c}^{(2)}
\end{array}\right\}\right) .
$$

Note that when $\Omega$ is set to zero in mass as well as stiffness matrices, the equations of IIE and SCE are reduced to those of the Euler-Bernoulli beam element.

\subsection{Evaluation of the elements for dynamic behaviour}

To evaluate how various elements predict the natural frequencies, the free vibration of a simply supported beam is studied. As per the Timoshenko beam theory, the natural frequencies of the simply supported beam can be calculated from the equations

$$
\begin{aligned}
& -\frac{\partial}{\partial x}\left(E I \frac{\partial \phi_{x}}{\partial x}\right)+G A K_{s}\left(\phi_{x}+\frac{\partial w}{\partial x}\right)+I_{2} \frac{\partial^{2} \phi_{x}}{\partial t^{2}}=0, \\
& -\frac{\partial}{\partial x}\left[G A K_{s}\left(\phi_{x}+\frac{\partial w}{\partial x}\right)\right]+I_{0} \frac{\partial^{2} w}{\partial t^{2}}=0 .
\end{aligned}
$$

For periodic motion, we assume that

$$
w(x, t)=W_{0}(x) \exp (-i \omega t), \quad \phi_{x}(x, t)=\Phi_{0}(x) \exp (-i \omega t),
$$

where $\omega$ denotes the frequency of natural vibration and $W_{0}$ and $\Phi_{0}$ denote the deflection and rotation mode shapes. Substitution of (93) into $(92 a, b)$ yields the equations

$$
\begin{aligned}
& -\frac{\mathrm{d}}{\mathrm{d} x}\left(E I \frac{\mathrm{d} \Phi_{0}}{\mathrm{~d} x}\right)+G A K_{s}\left(\phi_{x}+\frac{\mathrm{d} W_{0}}{\mathrm{~d} x}\right)-I_{2} \omega^{2} \Phi_{0}=0, \\
& -\frac{\mathrm{d}}{\mathrm{d} x}\left[G A K_{s}\left(\Phi_{0}+\frac{\mathrm{d} W_{0}}{\mathrm{~d} x}\right)\right]-I_{0} \omega^{2} W_{0}=0 .
\end{aligned}
$$

For a simply supported beam, we assume solution of the form,

$$
W_{0}(x)=W \sin (m \pi x / L), \quad \Phi_{0}(x)=\Phi \cos (m \pi x / L),
$$

which satisfy the boundary conditions $W_{0}(0)=0, W_{0}(L)=0$, where $L$ is the length of the beam. Substituting (94) into (93a,b), we obtain the eigenvalue problem

$$
\left[\begin{array}{cc}
\frac{m^{2} \pi^{2}}{L^{2}} E I+K_{s} G A-I_{2} \omega^{2} & \frac{m \pi}{L} K_{s} G A \\
\frac{m \pi}{L} K_{s} G A & \frac{m^{2} \pi^{2}}{L^{2}} K_{s} G A-I_{0} \omega^{2}
\end{array}\right]\left\{\begin{array}{l}
\Phi \\
W
\end{array}\right\}=\left\{\begin{array}{l}
0 \\
0
\end{array}\right\} .
$$

Setting the determinant of the coefficient matrix in (95) to zero, we obtain the frequency equation

$$
I_{2} \omega_{m}^{4}-K_{s} G A\left[\frac{m^{2} \pi^{2}}{L^{2}} \frac{I_{2}}{I_{0}}+m^{2} \pi^{2} \Omega_{L}+1\right] \omega_{m}^{2}+\left(\frac{m^{4} \pi^{4}}{L^{4}} \frac{E I}{I_{0}}\right) K_{s} G A=0,
$$


Table 1. Comparison of the pure flexural natural frequencies $\left(\times 10^{2}\right)$ obtained by the RIE elements with the exact frequencies of a simply supported beam.

\begin{tabular}{ccccccccc}
\hline & \multicolumn{3}{c}{ Linear elements } & & \multicolumn{3}{c}{ Quadratic elements } & \\
\cline { 2 - 3 }$m$ & $N=10^{\dagger}$ & $N=20$ & $N=40$ & & $N=5$ & $N=10$ & $N=20$ & Eq. (96) \\
\hline 1 & 0.28378 & 0.28121 & 0.28058 & & 0.28045 & 0.28037 & 0.28036 & 0.28036 \\
2 & 1.1234 & 1.0850 & 1.0758 & & 1.0775 & 1.0730 & 1.0727 & 1.07270 \\
3 & 2.4944 & 2.3184 & 2.2771 & & 2.3078 & 2.2666 & 2.2638 & 2.26356 \\
4 & 4.3792 & 3.8821 & 3.7693 & & 3.9282 & 3.7469 & 3.7335 & 3.73259 \\
5 & 6.7721 & 5.6986 & 5.4608 & & 5.7735 & 5.4291 & 5.3874 & 5.38433 \\
6 & - & 7.7157 & 7.2887 & & - & 7.2625 & 7.1600 & 7.15239 \\
7 & - & 9.9018 & 9.2119 & & - & 9.2226 & 9.0096 & 8.99313 \\
8 & - & 12.238 & 11.205 & & - & 11.303 & 10.910 & 10.8783 \\
\hline
\end{tabular}

${ }^{\dagger} N$ denotes the number of elements used in the total span of the beam.

where $\Omega_{L}=E I /\left(G A K_{s} L^{2}\right)$. Equation (96) can be used to determine the natural frequency $\omega_{m}$ for various values of $m$.

For pure shear mode, set $W$ to zero in (95) [or $I_{0} \rightarrow \infty$ in (96)] and obtain

$$
\omega_{m}^{s}=\left[\left(K_{s} G A / I_{2}\right)\left(m^{2} \pi^{2} \Omega+1\right)\right]^{1 / 2}, \quad \omega_{0}^{s}=\left(K_{s} G A / I_{0}\right)^{1 / 2} .
$$

It is clear from (96) that both shear deformation and rotatory inertia have the effect of reducing the frequency of natural vibration.

The first eight natural frequencies of pure flexural frequencies obtained using the reduced integration elements (RIE), interdependent interpolation element (IIE), and superconvergent element (SCE) of (89) are compared with the analytical results in tables 1,2, and 3, respectively. The following values of the parameters were used.

$$
E=1.0, \quad v=0.25, \quad K_{s}=\frac{5}{6}, \quad h=10.0, \quad A=10.0, \quad L=100.0 .
$$

For the same number of degrees of freedom, IIE predicts more accurate results than RIE (compare columns 2,3, and 4 of table 1 with columns 3,4, and 5 of table 2). Surprisingly, the quadratic RIE (with almost double the degrees of freedom), yields very accurate results in comparison with IIE. The superconvergent element (SCE) seems to underpredict the frequencies (i.e., frequencies converge to lower values than the exact). This might be due to the inconsistency of the mass matrix. It should be noted that a certain minimum number of elements are needed to predict a desired number of lowest frequencies. In general, the

Table 2. Comparison of the pure flexural natural frequencies $\left(\times 10^{2}\right)$ obtained by the IIE elements with the exact frequencies of a simply supported beam.

\begin{tabular}{cccccc}
\hline$m$ & $N=5$ & $N=10$ & $N=20$ & $N=40$ & Eq.(96) \\
\hline 1 & 0.28050 & 0.28039 & 0.28037 & 0.28037 & 0.28036 \\
2 & 1.0801 & 1.0743 & 1.0731 & 1.0728 & 1.07270 \\
3 & 2.3303 & 2.2783 & 2.2671 & 2.2644 & 2.26356 \\
4 & 4.0087 & 3.7975 & 3.7483 & 3.7365 & 3.73259 \\
5 & - & 5.5756 & 5.4310 & 5.3959 & 5.38433 \\
6 & - & 7.5920 & 7.2610 & 7.1794 & 7.15239 \\
7 & - & 9.8401 & 9.2082 & 9.0466 & 8.99313 \\
8 & - & 12.280 & 11.258 & 10.973 & 10.8783 \\
\hline
\end{tabular}


Table 3. Comparison of the pure flexural natural frequencies $\left(\times 10^{2}\right)$ obtained by the SCE elements with the exact frequencies of a simply supported beam.

\begin{tabular}{lclllc}
\hline$m$ & $N=5$ & $N=10$ & $N=20$ & $N=40$ & Eq. (97) \\
\hline 1 & 0.28040 & 0.28033 & 0.28031 & 0.28031 & 0.28036 \\
2 & 1.0750 & 1.0708 & 1.0700 & 1.0698 & 1.07270 \\
3 & 2.2834 & 2.2472 & 2.2399 & 2.2382 & 2.26356 \\
4 & 3.7869 & 3.6657 & 3.6349 & 3.6275 & 3.73259 \\
5 & - & 5.5756 & 5.1163 & 5.0957 & 5.38433 \\
6 & - & 8.1943 & 6.5812 & 6.5377 & 7.15239 \\
7 & - & 9.4363 & 7.9633 & 7.8876 & 8.99313 \\
8 & - & 10.306 & 9.2257 & 9.1117 & 10.8783 \\
\hline
\end{tabular}

frequencies predicted with a fixed number of elements will not always be pure bending frequencies; the set may also contain pure shear frequencies (as is the case here when 10 linear RIE elements or 5 IIE elements are used).

Table 4 contains the first ten pure shear frequencies obtained using RIE (linear elements) and IIE. The performance of RIE is superior to IIE in predicting the pure shear frequencies. IIE yields frequencies which converge slowly as the mesh is refined. The convergences of both RIE and IIE are not monotonic, especially for higher frequencies. Table 5 contains results obtained using RIE with quadratic elements. The convergence is very good. The superior performance of RIE over IIE is due to the fact that the stiffness matrix of IIE is based on constant shear strain representation.

\section{Summary}

A complete set of finite element models of the Timoshenko beam theory is presented using displacement and assumed strain-displacement approaches. Alternative finite element formulations of the Timoshenko beam theory which give the $4 \times 4$ superconvergent stiffness matrix are presented using assumed strain-displacement formulation of the conventional Timoshenko beam theory and the displacement formulation of a modified form of the Timoshenko beam theory in which the total transverse deflection is decomposed into bending and shear deflections. In the latter case, the final equations were recast only in terms of the

Table 4. Comparison of the pure shear natural frequencies obtained by various elements with the exact frequencies of a simply supported beam.

\begin{tabular}{llllllll}
\hline$m$ & Eq. (97) & RIE(10) & RIE(20) & RIE(40) & IIE(10) & IIE(20) & IIE(40) \\
\hline 0 & 0.20000 & 0.20000 & 0.20000 & 0.20000 & 0.22771 & 0.20801 & 0.20206 \\
1 & 0.20245 & 0.20165 & 0.20225 & 0.20240 & 0.22789 & 0.21045 & 0.20454 \\
2 & 0.20964 & 0.20669 & 0.20892 & 0.20946 & 0.22841 & 0.21764 & 0.21179 \\
3 & 0.22109 & 0.21534 & 0.21976 & 0.22077 & 0.22917 & 0.22921 & 0.22341 \\
4 & 0.23620 & 0.22797 & 0.23443 & 0.23578 & 0.23007 & 0.24465 & 0.23880 \\
5 & 0.25431 & 0.24495 & 0.25259 & 0.25391 & 0.23099 & 0.26342 & 0.25737 \\
6 & 0.27483 & 0.26639 & 0.27394 & 0.27466 & 0.23185 & 0.28502 & 0.27856 \\
7 & 0.29726 & 0.29150 & 0.29824 & 0.29759 & 0.23257 & 0.30899 & 0.30194 \\
8 & 0.32119 & 0.31742 & 0.32532 & 0.32235 & 0.23311 & 0.33492 & 0.32713 \\
9 & 0.34633 & 0.33823 & 0.35505 & 0.34870 & 0.23344 & 0.36246 & 0.35388 \\
\hline
\end{tabular}


Table 5. Comparison of the pure shear natural frequencies obtained by various elements with the exact frequencies of a simply supported beam.

\begin{tabular}{lcccccccc}
\hline Mesh & $m=0$ & $m=1$ & $m=2$ & $m=3$ & $m=4$ & $m=5$ & $m=6$ & $m=7$ \\
\hline Exact & 0.20000 & 0.20245 & 0.20964 & 0.22109 & 0.23620 & 0.25431 & 0.27483 & 0.29726 \\
$\mathrm{Q}(5){ }^{\dagger}$ & 0.20000 & 0.20242 & 0.20926 & 0.21957 & 0.23224 & 0.26458 & 0.27837 & 0.30496 \\
$\mathrm{Q}(10)$ & 0.20000 & 0.20245 & 0.20962 & 0.22101 & 0.23602 & 0.25403 & 0.27459 & 0.29735 \\
$\mathrm{Q}(20)$ & 0.20000 & 0.20245 & 0.20964 & 0.22109 & 0.23619 & 0.25429 & 0.27482 & 0.29728 \\
\hline
\end{tabular}

total deflection and rotation at the nodes, and the resulting finite element stiffness matrix is $4 \times 4$ for (bending only). The developments are then extended to the dynamic case. Numerical results of natural frequencies indicate that the reduced integration model (RIE) or its equivalent predict both flexural and pure shear frequencies accurately, provided a sufficient number of elements are used. The interdependent interpolation element (IIE) predicts flexural frequencies accurately but exhibits slow convergence, and it is poor in predicting pure shear frequencies. This is possibly due to the fact that IIE is based on approximation functions derived from a constant state of shear strain. The constant state of shear strain is statically correct but it is incorrect for dynamic problems, as can be seen from $(91 \mathrm{a}, \mathrm{b})$ or $(93 \mathrm{a}, \mathrm{b})$.

The research reported herein is supported by the Oscar S. Wyatt Endowed Chair. It is a pleasure to acknowledge the discussions on the topic with Dr. Gangan Prathap of the National Aerospace Laboratories, Bangalore, India and with Professor A V Krishna Murty of the Aerospace Engineering Department, Indian Institute of Science, Bangalore, India.

\section{References}

Anderson R A 1953 Flexural vibrations in uniform beams according to the Timoshenko theory. J. Appl. Mech. 20: 504-510

Babu C R, Prathap G 1986 A linear thick curved beam element. Int. J. Numer. Methods Eng. 23: 1313-1328

Bhashyam G R, Gallagher R H 1984 An approach to the inclusion of transverse shear deformation in finite element plate bending analysis. Comput. Struct. 19: 35-40

Chow T S 1971 On the propagation of flexural waves in an orthotropic laminated plate and its response to an impulsive load. J. Composite Materials 5: 306-318

Friedman Z, Kosmatka J B 1993 An improved two-node Timoshenko beam finite element. Comput. Struct. 47: 473-481

Gere J M, Weaver W Jr 1965 Analysis of framed structures (New York: Van Nostrand) pp. 428-430

Huffington N J 1963 Response of elastic columns to axial pulse loading. AIAA J. 1: 2099-2104

Krishna Murty A V 1970 Vibrations of short beams. AIAA J. 8: 34-38

Lim S P, Lee K H, Chow S T, Senthilnathan N R 1988 Linear and nonlinear bending of sheardeformable plates. Comput. Struct. 30: 945-952

Miklowitz J 1953 Flexural wave solutions of coupled equations representing the more exact theory of bending. J. Appl. Mech. 20: 511-514 
Miklowitz J 1960 Flexural stress wave in an infinite elastic plate due to a suddenly applied concentrated transverse load. J. Appl. Mech. 27: 681-689

Oden J T, Reddy J N 1982 Variational methods in theoretical mechanics 2nd edn (Berlin: SpringerVerlag)

Prathap G, Babu C R 1986 Field-consistent strain interpolation for the quadratic shear flexible beam element. Int. J. Numer. Methods Eng. 23: 1973-1984

Prathap G, Bhashyam G R 1982 Reduced integration and the shear flexible beam element. Int. J. Numer. Methods Eng. 18: 195-210

Przemieniecki J S 1968 Theory of matrix structural analysis (New York: McGraw-Hill)

Rakowski J 1991 A critical analysis of quadratic beam finite elements. Int. J. Numer. Methods Eng. 31: 949-966

Reddy J N 1987 A small strain and moderate rotation theory of laminated anisotropic plates. $J$. Appl. Mech. 54: 623-626

Reddy J N 1993 An introduction to the finite element method 2nd edn (New York: McGraw-Hill) pp. $177-187$

Reddy J N 1997 On locking-free shear deformable beam elements. Comput. Methods Appl. Mech. Eng. 149: 113-132

Reddy J N 1999 Theory and analysis of elastic plates (Philadelphia: Taylor \& Francis) pp 484-493

Reddy J N, Wang C M, Lam K Y 1997 Unified finite elements based on the classical and shear deformation theories of beams and axisymmetric circular plates. Commun. Numer. Methods Eng. 13: 495-510

Senthilnathan N R, Lim S P, Lee K H, Chow S T 1988 Vibration of laminated orthotropic plates using a simplified higher-order deformation theory. Composite Struct. 10: 211-229

Shi G, Voyiadjis G Z 1991 Simple and efficient shear flexible two-node arch/beam and four-node cylindrical shell/plate finite elements. Int. J. Numer. Methods Eng. 31: 759-776 